\title{
Film Cooling Effectiveness Data for Simple Injection Geometries: A Collection of Three-Axis Plots
}

\author{
L. D. STONE and R. J. GOLDSTEIN \\ Heat Transfer Laboratory, Mechanical Engineering Department, University of Minnesota, Minneapolis, MN 55455
}

\begin{abstract}
A three-axis plot of laterally averaged film cooling effectiveness versus blowing rate and versus dimensionless distance downstream is a sufficiently compact representation of film cooling effectiveness data for injection through a row of holes or two rows of holes to enable easy, global comparisons among a variety of data sets. By plotting data from several film cooling studies conducted at the University of Minnesota, the dominant roles played by density ratio, hole spacing, and surface curvature become apparent. It is also seen that a slot-plate prediction/correlation from two-dimensional film cooling studies makes an excellent standard of comparison for all film cooling studies, even though the more commonly encountered (two-axis) comparisons using standardized nondimensional parameters do not usually suggest this.
\end{abstract}

Key Words: Convective heat transfer; Film cooling; Gas turbines; Boundary layers; Jets in crossflow; Mixing

\section{THEORETICAL BASIS}

A SIMPLE analysis which produces a surprisingly good prediction of film cooling effectiveness for tangential-slot injection along flat plates is outlined by Goldstein [1971] (similar "two-dimensional" models earlier postulated by others are also discussed therein). Essentially the coolant film is modeled as a self-similar boundary layer which is "filled" with pure coolant at injection (note that this defines an effective starting length back of injection) and which entrains hot, mainstream fluid as it grows downstream (Figure 1). The three, main, simplifying assumptions are 1) that this fictitious boundary layer is everywhere well mixed, hence isothermal at each position $x ; 2)$ that these two fluids mix adiabatically, in other words, no heat transfers to or from the wall nor across the edge of the boundary layer; and 3 ) that the resulting temperature of this fictitious boundary layer at any position $x$ is identically the adiabatic wall temperature. Describing the entrainment rate of freestream fluid according to "textbook" predictions for the growth of a constant-properties, $1 / 7$ th-profile, flat-plate, turbulent boundary layer, and further assuming constant and equal specific heats, it predicts:

$$
\eta=\{1+0.249 \zeta\}^{-4 / 5}
$$

where

$$
\zeta=\left\{\frac{x}{M s}\right\}\left\{\operatorname{Re}_{2} \frac{\mu_{2}}{\mu_{\infty}}\right\}^{-1 / 4}
$$

This formula successfully collapses data for tangentialslot injection along flat plates, and will be referred to in this note as the slot-plate correlation.

Note that the correlation indicates that the film cooling effectiveness is a function of four dimensionless parameters: blowing rate, dimensionless distance downstream, the freestream-to-injectant viscosity ratio, and the injection Reynolds number. The injection Reynolds number is itself however, a function of blowing rate. To isolate the effect of blowing rate, eq. [2a] is rewritten as:

$$
\zeta=\left\{\frac{x}{M s}\right\}\left\{\frac{\rho_{2} u_{2} s}{\mu_{\infty}}\right\}^{-1 / 4}
$$

but $\rho_{2} u_{2}=\rho_{\infty} u_{\infty} M$ so

$$
\zeta=\left\{\frac{x}{s}\right\}\{M\}^{-5 / 4}\left\{\frac{\rho_{\infty} u_{\infty} s}{\mu_{\infty}}\right\}^{-1 / 4}
$$

Now only three dimensionless parameters appear in the definition of $\zeta$ (the final term, of course, is the freestream Reynolds number based on the slot height). According 


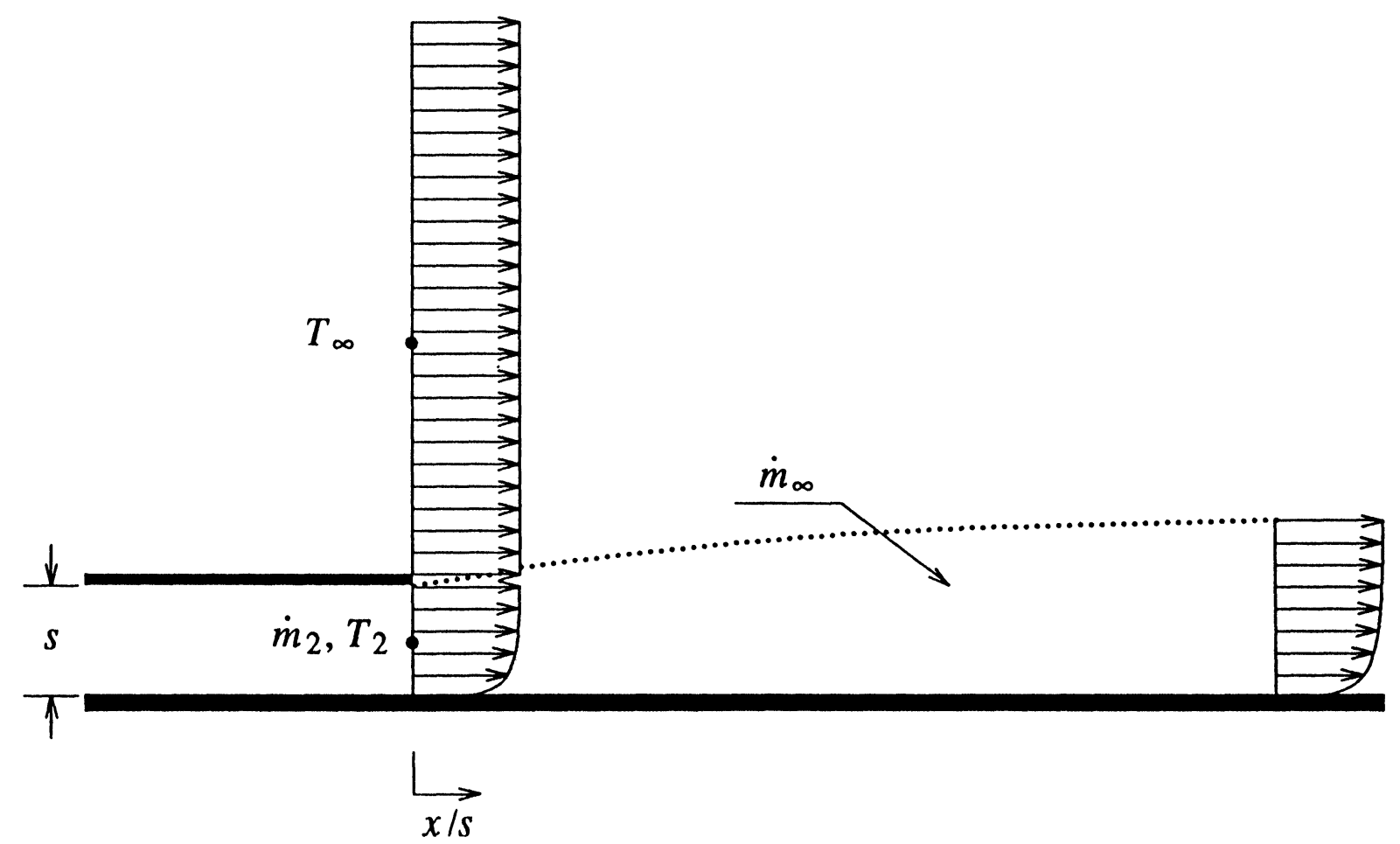

FIGURE 1 The boundary layer entrainment model of film cooling.

to the prediction, then, for a fixed freestream Reynolds number the film cooling effectiveness is a function only of blowing rate and dimensionless distance downstream. Put another way, equation 1 describes surfaces in $\eta-x / s-M$ space, each corresponding to a given freestream Reynolds number.

Before proceeding further, a minor modification is made to eq. [2]. Many film cooling studies feature injection from a row of holes spaced three diameters center-to-center, for which an equivalent slot height can be defined as:

$$
s_{e q v}=\frac{\pi D}{12}
$$

With this final substitution, the boundary-layer entrainment model predicts:

$$
\tilde{\eta}=\{1+0.249 \zeta\}^{-4 / 5}
$$

where

$$
\zeta=\left\{\frac{x}{D}\right\}\left\{\frac{\pi M}{12}\right\}^{-5 / 4}\left\{\operatorname{Re}_{D}\right\}^{-1 / 4}
$$

\section{PRESENTATION OF THREE-AXIS PLOTS}

Figure 2 shows the slot-plate correlation when the freestream Reynolds number based on the injection hole diameter is 10,000 . Film cooling effectiveness is zero at a blowing rate of zero but increases rapidly as the blowing rate is increased from zero, asymptotically approaching $100 \%$ at infinity. In the $x$-direction, effectiveness is $100 \%$ at injection but falls off sharply as one moves downstream, gradually approaching zero at infinity. Stretch a net over these asymptotic boundary conditions and you get the smoothly-curving surface shown in Figure 2, which shows quite clearly that the rate of fall of effectiveness in the streamwise direction must decrease with increased blowing rate, that the rate of rise in effectiveness with blowing rate must decrease with increased distance downstream, and so on.

Figure 2 makes a useful standard for comparison. With few exceptions, laterally averaged effectiveness data for discrete-holes injection either fall on or below the surface of Figure 2 (this fact is easily anticipated, actually, if one agrees that a tangential slot is the injection geometry of choice). 
TABLE I

Summary of film cooling studies considered in this note

\begin{tabular}{|c|c|c|c|c|c|c|c|}
\hline Study & Wall & $\begin{array}{l}\text { No. Rows } \\
\text { of Holes }\end{array}$ & $\begin{array}{c}\text { Primary } \\
\text { Variable } \\
\text { of Interest }\end{array}$ & $\operatorname{Re}_{D}$ & $\rho_{2} / \rho_{\infty}$ & $M$ & $x / D$ \\
\hline Goldstein [1971] & flat plate & - & a & $10,000^{b}$ & c & $0-2^{b}$ & $8-40^{b}$ \\
\hline Pedersen [1971] & flat plate & 1 & $\rho_{2} / \rho_{\infty}$ & $11,000^{d}$ & $0.75-4.2$ & $0.2-2.0$ & $4-110$ \\
\hline Jabbari [1973] & flat plate & 2 & $K$ & 11,000 & $0.84,1$ & $0.2-1.5$ & $6-82$ \\
\hline Schwarz [1986] & $\begin{array}{l}\text { convex } \\
\text { concave }\end{array}$ & $\begin{array}{l}1 \\
1\end{array}$ & $2 r / D$ & $\begin{array}{l}8,000 \\
9,000\end{array}$ & $0.95,2$ & $\begin{array}{l}0.4-2.2 \\
0.3-2.5\end{array}$ & $\begin{array}{l}3-44^{\mathrm{e}} \\
6-45^{\mathrm{e}}\end{array}$ \\
\hline Ito [1973] & $\begin{array}{c}\text { suction side } \\
\text { pressure side }\end{array}$ & $\begin{array}{l}1 \\
1\end{array}$ & blade geometry & $\begin{array}{r}3,500 \\
900\end{array}$ & $0.95,2$ & $\begin{array}{l}0.2-2.0 \\
0.2-2.0\end{array}$ & $\begin{array}{l}4-56 \\
4-49\end{array}$ \\
\hline Kornblum [1981] & $\begin{array}{l}\text { suction side } \\
\text { pressure side }\end{array}$ & $\begin{array}{l}2 \\
2\end{array}$ & second row & $\begin{array}{r}3,000 \\
750\end{array}$ & $0.95,2$ & $\begin{array}{l}0.25-2.0 \\
0.25-2.0\end{array}$ & $\begin{array}{l}7-62 \\
6-54\end{array}$ \\
\hline
\end{tabular}

a Analysis

b Assumed for the sake of comparison

c No explicit dependence

d 20,000 for $M=0.2$

e For $2 r / D \approx \pm 90$

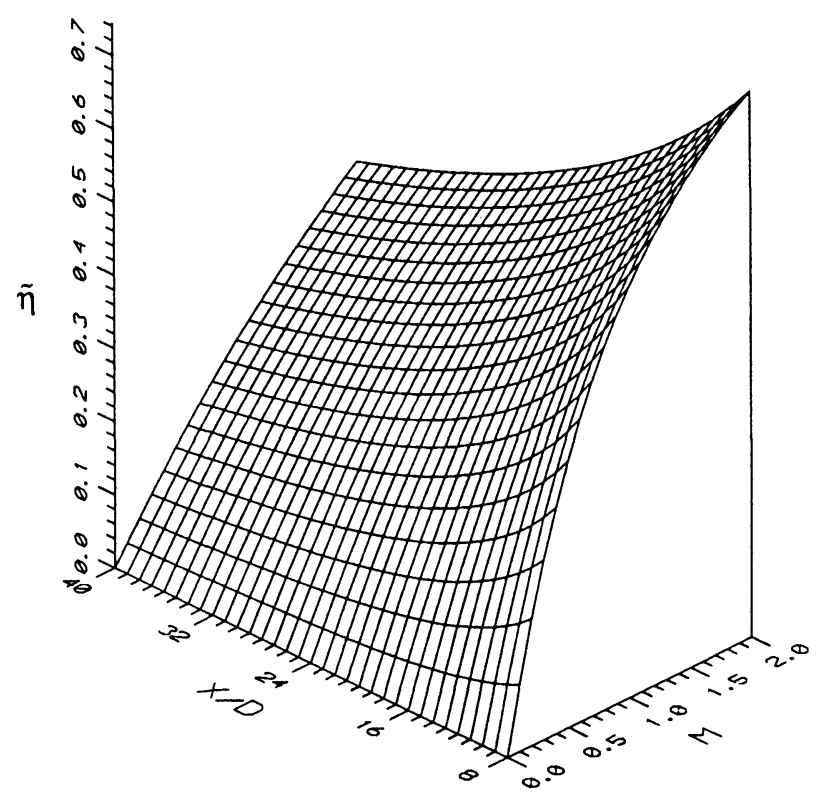

FIGURE 2 Effectiveness surface representation of the boundary-layer entrainment model of film cooling presented in Goldstein [1971] (for case: $\left.\operatorname{Re}_{D}=10,000\right)$.

In many of the figures that follow, an outline of Figure 2 has been superimposed, for ease of comparison. Strictly speaking, eq. [4] predicts a unique surface for a given $\operatorname{Re}_{D}$, which means that a separate surface should be plotted for each study under consideration. Since the freestream Reynolds number is predicted to affect the film cooling effectiveness only to the one-fourth power, however, and since Table I shows that many of the studies considered here were run at Reynolds numbers close to 10,000 , this value of Reynolds number is generally used herein.

Figure 3 shows a fit to the laterally averaged effectiveness data of Pedersen [1971] (see also Pedersen et al. [1977]) for discrete-holes injection along a flat plate. At a density ratio of one (Figure 3a) and very low blowing rates his data follow the form of Figure 2, but drop sharply from this surface at a blowing rate of about 0.5 . The jets have lifted off from the wall, and since the mainflow is able to move around and under the jets, the film cooling effectiveness at the wall is considerably reduced. With increased blowing the effectiveness slowly increases again, at least at large $x / D$, but is still far below the slot-plate correlation.

Pedersen's data for a density ratio of two are shown in Figure $3 b$. The surface is geometrically similar to that of Figure $3 \mathrm{a}$; the main difference in the two surfaces is that lift-off has been delayed to a higher blowing rate by the use of a higher density ratio. This trend continues through a density ratio of four, as shown in Figure $3 \mathrm{c}$. These figures clearly show that the main effect of increased density ratio is the delay of lift-off to higher blowing rates, with higher values of film cooling effectiveness being associated with the lift-off blowing rate. More succinctly, the falling away of the data from the slot-plate surface of Figure 2 is delayed to a higher blowing rate by the use of a higher density ratio. (In cooling applications, of course, a higher density ratio implies a cooler injection temperature; use of cooler injectant, therefore, is doubly beneficial.)

Figure 4 shows the data from Jabbari [1973] (see also Jabbari and Goldstein [1978]) for injection from a double 


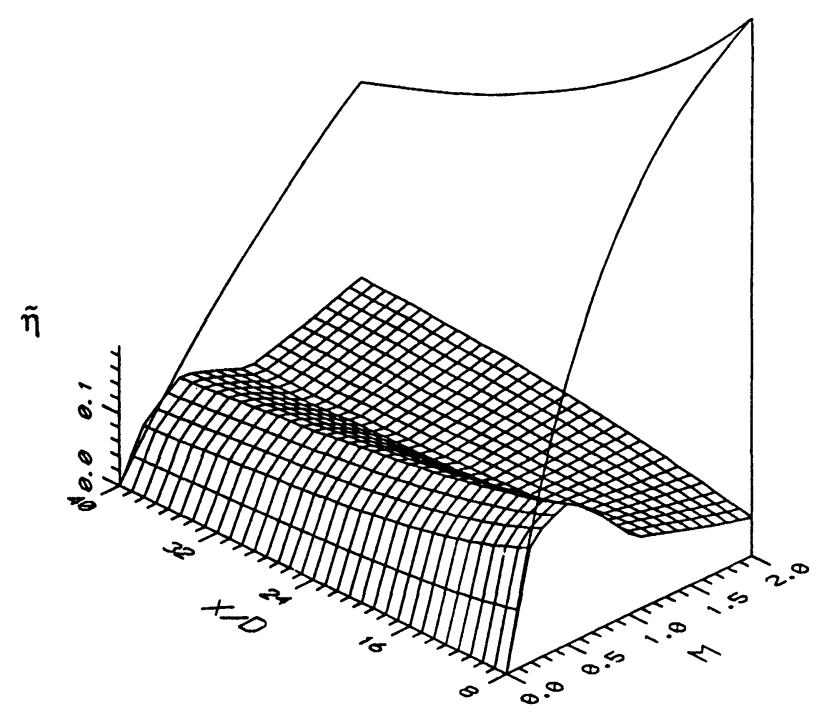

(a) $\rho_{2} / \rho_{\infty}=0.96$

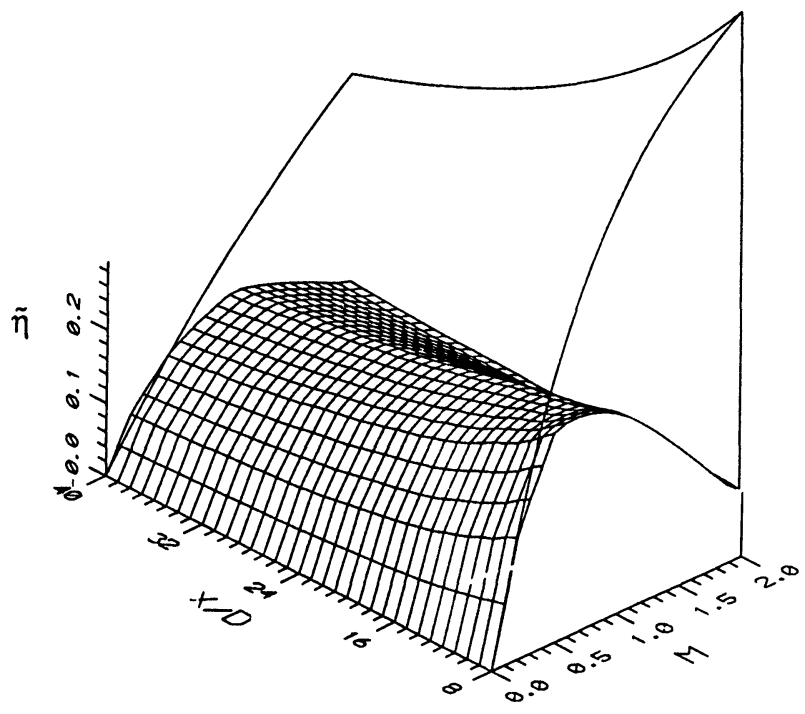

(b) $\rho_{2} / \rho_{\infty} \approx 2.0$

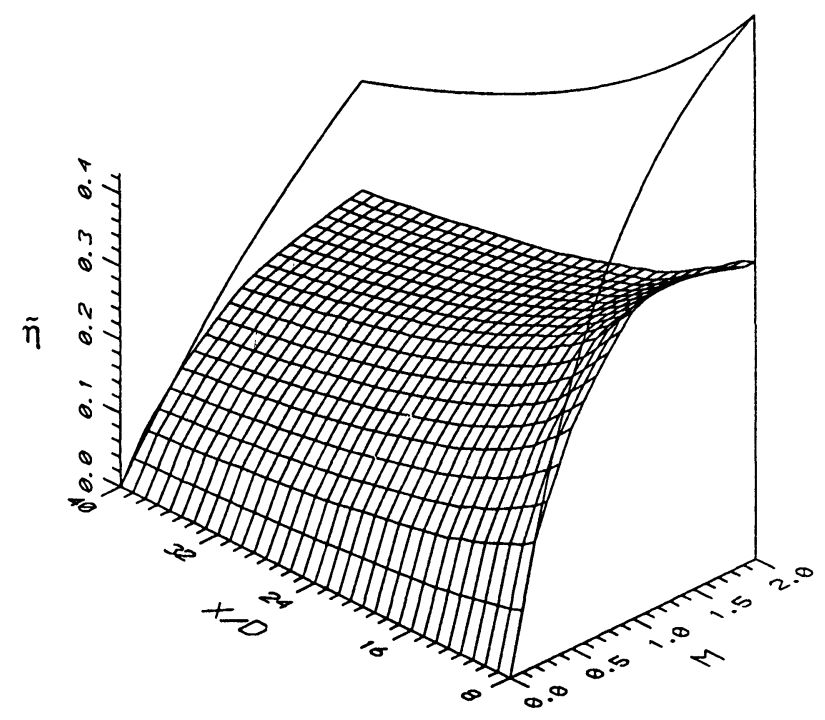

(c) $\rho_{2} / \rho_{\infty} \approx 4.17$

FIGURE 3 Effectiveness surface representation of the data of Pedersen [1971] (single row of holes, flat plate; reference surface from Figure 2).

row of holes (staggered) along a flat plate at a density ratio of about 0.84 . Note that the blowing rate in Figure 4 is actually twice Jabbari's reported blowing rate, such that this comparison of double-row data with single-row data is made based on an equal mass rate of coolant injection per unit span.

Doubling the number of holes has two effects. First, lift-off is delayed to a higher blowing rate; doubling the number of holes effectively halves the injection velocity for a given mass flow of injectant per unit span, which obviously should increase the effective blowing rate at which lift-off would occur. Recall that a similar effect occurs when the density ratio is increased; doubling the density ratio also halves the mean velocity of the jets.

The second effect of doubling the number of holes is seen by contrasting Pedersen's and Jabbari's data (Figures $3 a, 4)$. In the latter study the effectiveness does not drop off after jet lift-off, but reaches a plateau instead. Apparently the closer spacing of the injection holes limits the ability of the mainstream fluid to get around and under the jets. From 


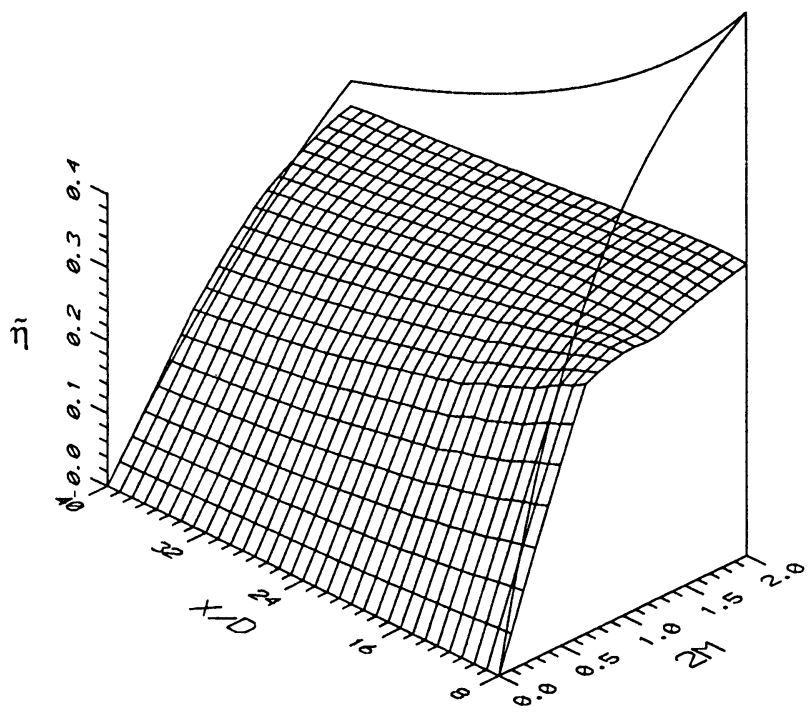

FIGURE 4 Effectiveness surface representation of the data of Jabbari [1973] (double row of holes, flat plate, $\rho_{2} / \rho_{\infty} \approx 0.84$; reference surface from Figure 2).

the standpoint of film cooling effectiveness, Figure $3 \mathrm{a}$ and 4 emphatically show that the use of a double row of holes is a better design than a single row of holes, at least for injection along flat plates at moderate to high blowing rates.

Figure 5 shows a fit to the data from Schwarz [1986] (see also Schwarz et al. [1991]) for row-of-holes injection along a convex wall at a density ratio of one and two. At the lower density ratio (Figure 5a) before lift-off the effectiveness is actually a bit higher than that predicted by the reference surface. This is an effect of the rather strong cross-stream pressure gradient on the convex wall which works to "push" low-velocity coolant against the wall. It also reduces the boundary layer entrainment rate, as described by Meroney and Bradshaw [1975]. At blowing rates beyond lift-off, however, the benefit from the crossstream pressure gradient is lost; lift-off is quite severe, and the resulting effectiveness levels comparable to Pedersen's flat-plate data at similar blowing rates and density ratios. At a density ratio of two (Figure $5 b$ ), the primary effect of increased density ratio is again to delay lift-off to a higher blowing rate.

Schwarz's data for injection along a concave wall (Figure 6) follow a noticeably different pattern than what has been considered so far. At a density ratio of one (Figure 6a), effects of jet lift-off are still detected, though barely, close to injection, but even at low blowing rates the effectiveness is well below the slot-plate prediction. Far downstream the effectiveness increases almost linearly with blowing rate over the entire range of measurement. Apparently, the mixing within the concave-wall boundary layer is so thorough in these regions that events at injection are of little consequence; what matters far downstream is the mass of coolant that has been injected into the boundary layer. Even doubling the density ratio yields a nearly identical effectiveness surface in the far downstream regions

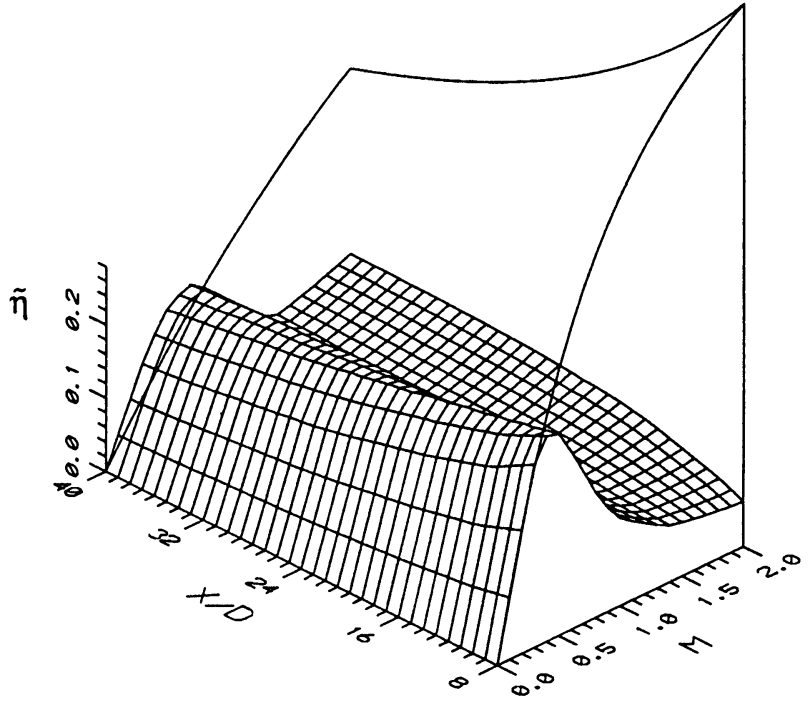

(a) $\rho_{2} / \rho_{\infty} \approx 0.95$

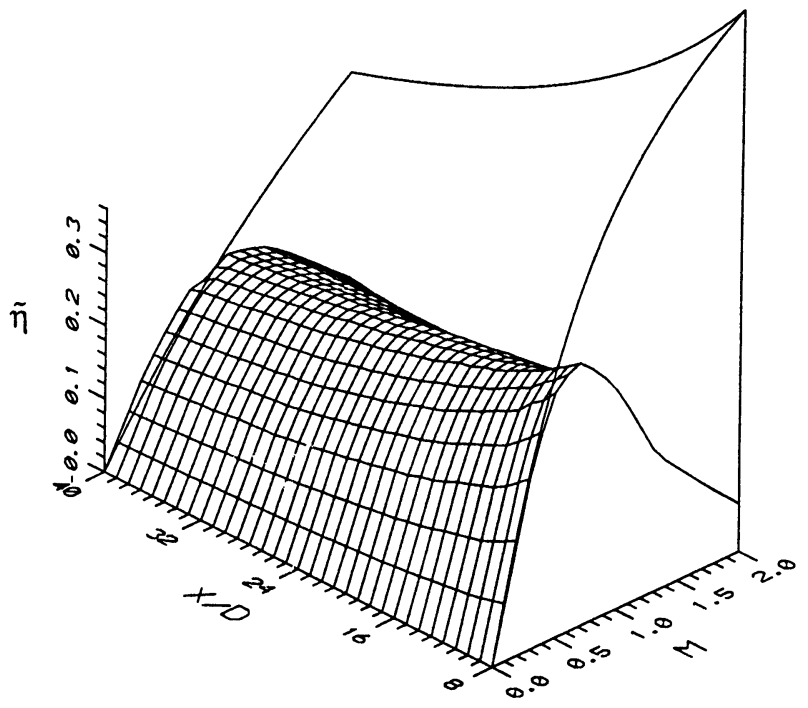

(b) $\rho_{2} / \rho_{\infty} \approx 2.0$

FIGURE 5 Effectiveness surface representation of the data of Schwarz [1986] (single row of holes, convex wall, $2 r / D \approx+94 ;$ reference surface from Figure 2). 


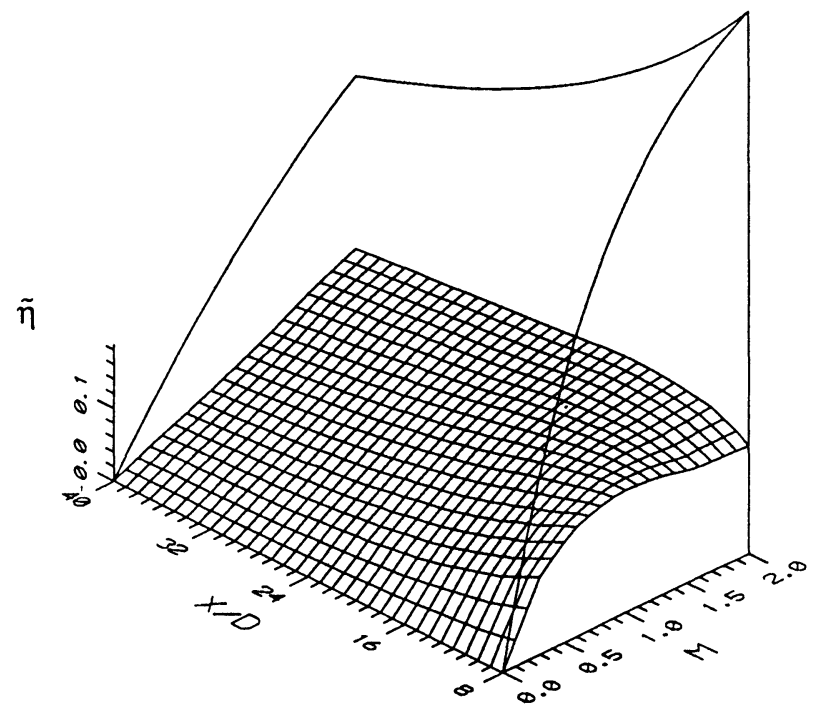

(a) $\rho_{2} / \rho_{\infty} \approx 0.95$

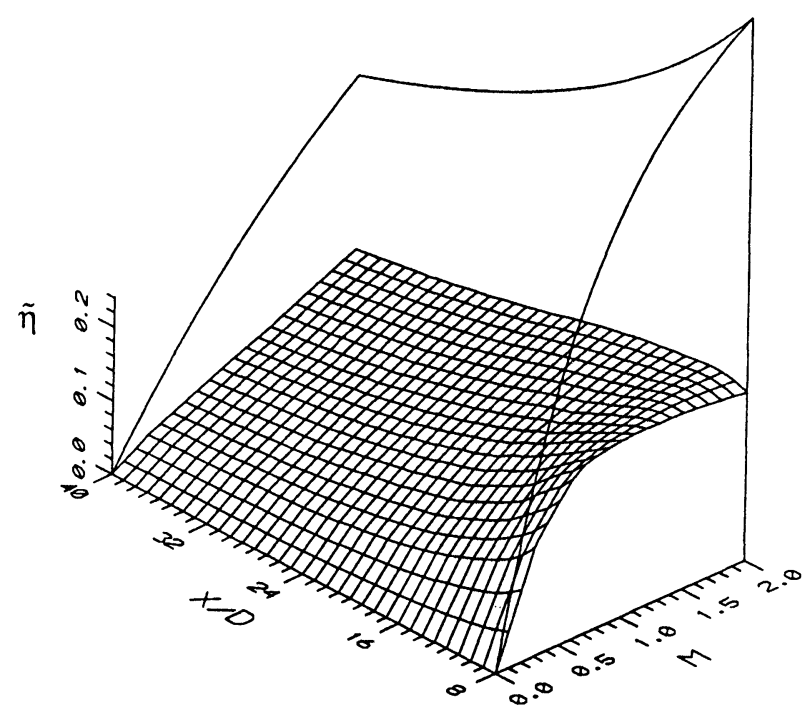

(b) $\rho_{2} / \rho_{\infty} \approx 2.0$

FIGURE 6 Effectiveness surface representation of the data of Schwarz [1986] (single row of holes, concave wall, $2 r / D \approx-89 ;$ reference surface from Figure 2).

(Figure 6b), although the characteristic delay in the lift-off blowing rate still registers near injection at the higher density ratio (one expects, of course, that if data closer than eight diameters from injection could have been included in these figures, the effect of jet lift-off and its delay would show up more dramatically).

Why are the effectiveness levels in Schwarz's concavewall data far below that of the slot-plate prediction? If the coolant mixes so thoroughly into the concave-wall boundary layer that the effectiveness downstream rises almost linearly with blowing rate, then one expects that the slope of effectiveness versus blowing rate at such a position would depend greatly on the boundary layer thickness. Whereas the two-dimensional analysis assumes a boundary layer thickness roughly equal to the slot height, Schwarz's boundary layer is actually almost 20 equivalent slot heights in thickness. The coolant in Schwarz's concave-wall data dilutes with more mainstream fluid than the slot-plate correlation assumes.

Figures 7a, b show the data of Ito [1973] (see also Ito et al. [1978]) for row-of-holes injection along a twodimensional turbine blade. Figure 2 has not been superimposed on Ito's data since his freestream Reynolds numbers were significantly less than 10,000 (Table I). Rather, slot-plate prediction surfaces have been recomputed for Reynolds numbers of 3,200 and 800 and outlines of these surfaces have been superimposed on the corresponding data of Ito (and on Kornblum's data, which follow in Figure 8).
The plot for the suction (convex) side (Figure 7a) is hardly surprising - it resembles in quality the convex-wall data of Schwarz. Before lift-off the effectiveness rises noticeably above the two-dimensional model surface (recall that there is a cross-stream pressure gradient into the wall), dropping off sharply after reaching a lift-off blowing rate of about one-half. The pressure (concave) side data (Figure 7b), as expected, resemble more closely the concave-wall data of Schwarz: lift-off effects are much less pronounced than on the suction (convex) side and confined close to injection, while the effectiveness increases monotonically with blowing rate in the far-downstream regions. However, Ito's pressure-side effectiveness surface is probably better described as a cross between Schwarz's convex-wall and concave-wall results. The differences between the concave-wall data of Schwarz and the pressureside data of Ito could be due to a difference in approach boundary layer thickness. It could also be partly a result of the non-constant curvature of a turbine blade, which would affect the streamwise development of the boundary layer.

The experiments of Kornblum [1981] (see also Goldstein et al. [1982]) were essentially the same as Ito's, except Kornblum used two rows of injection holes. The suction-side effectiveness surface (Figure 8a) is reminiscent of Jabbari's data (Figure 4): lift-off is delayed to a blowing rate of about one or so, and as the blowing rate is increased from one the effectiveness plateaus rather than drops. Significantly, whereas Jabbari's data lies on 


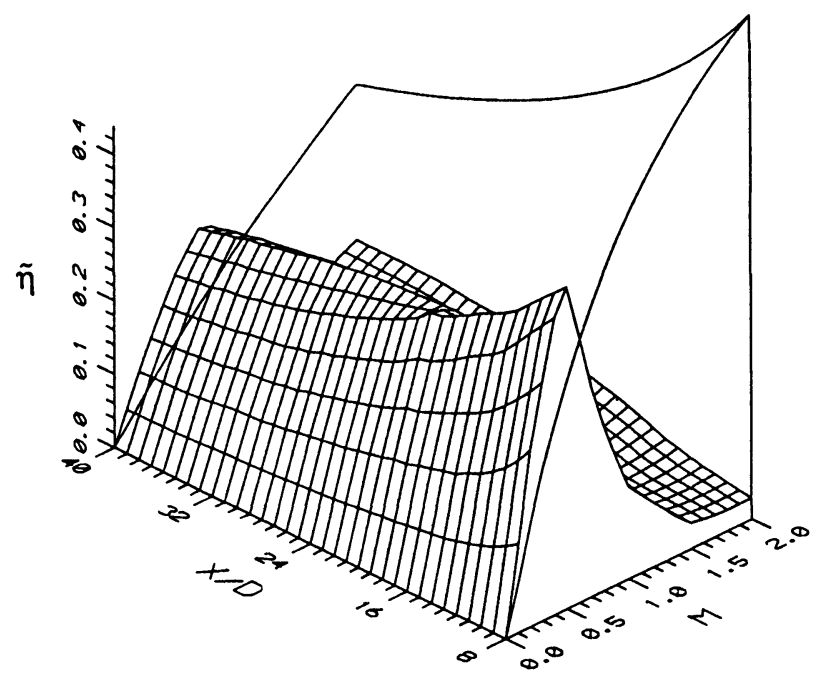

(a) Suction Side (Convex)

(Reference Surface for $\operatorname{Re}_{D}=3200$ )

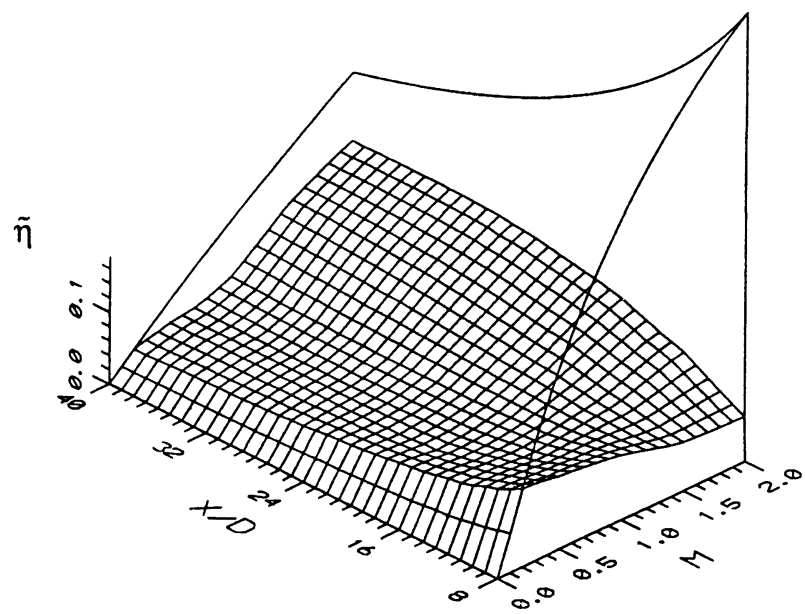

(b) Pressure Side (Concave)

(Reference Surface for $\mathrm{Re}_{D}=800$ )

FIGURE 7 Effectiveness surface representation of the data of Ito [1973] (single row of holes, linear cascade, $\rho_{2} / \rho_{\infty} \approx 0.95$ ).

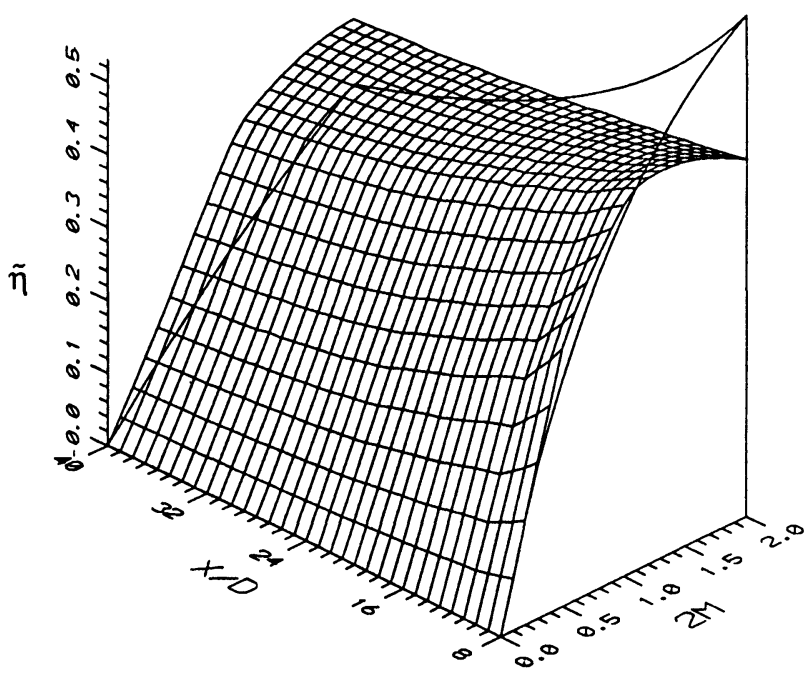

(a) Suction Side (Convex)

(Reference Surface for $\operatorname{Re}_{D}=3200$ )

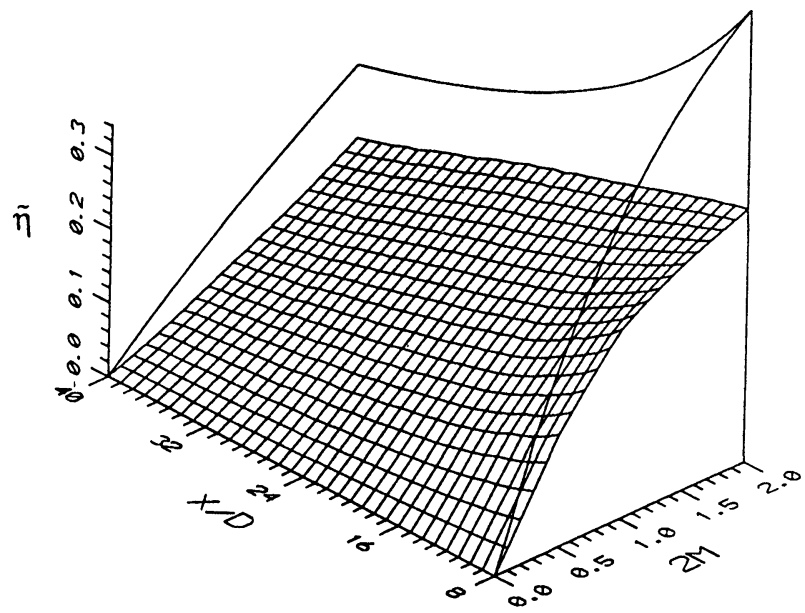

(b) Pressure Side (Concave)

(Reference Surface for $\operatorname{Re}_{D}=800$ )

FIGURE 8 Effectiveness surface representation of the data of Kornblum [1981] (double row of holes, linear cascade, $\rho_{2} / \rho_{\infty} \approx 0.96$ ).

or below the slot-plate surface, Kornblum's cuts through far downstream. Apparently the rate of entrainment of freestream fluid into his suction-side boundary layer is much lower than what obtains in flat-plate boundary layers (which, again, is an expected result on convex surfaces). In any case, a double row of holes is shown to be superior to a single row of holes, along pressure-side surfaces as well as along flat plates.
Kornblum's pressure-side data (Figure $8 \mathrm{~b}$ ) are Jabbarilike near injection and Schwarz-concave-like far downstream (as with Jabbari's data, the blowing rates used in Figure 8 are actually twice those reported by Kornblum). Near injection, the use of a double row of holes prevents the decline in effectiveness that occurs for single rows of holes at blowing rates greater than the lift-off value. Farther downstream, the increase of effectiveness 


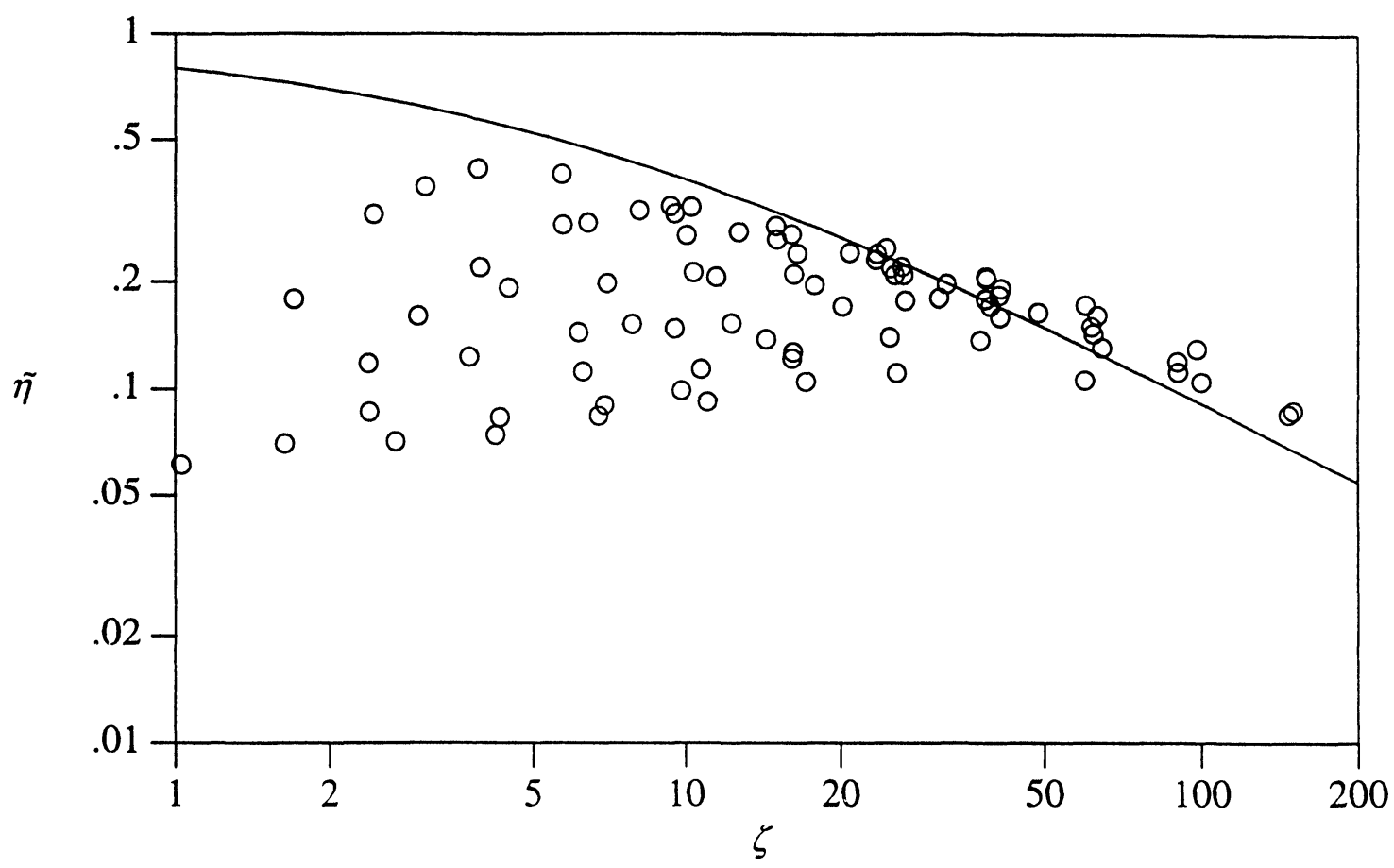

FIGURE 9 Comparison of the convex-wall data of Schwarz [1986] for row-of-holes injection at density ratios of approximately one and two to the slot-injection correlation presented in Goldstein [1971] using two-axis format.

with blowing rate is nearly linear, as was the case for Schwarz's concave-wall data. As with Schwarz's data, the effectiveness is well beneath the two-dimensional prediction, though by a smaller fraction than Schwarz's data. It may be that the relative boundary layer (hence, cooling film) thickness is smaller on Kornblum's pressure (concave) surface than on Schwarz's concave wall, which results in lower dilution of the coolant with mainstream fluid and which, in turn, results in higher levels of film cooling effectiveness on Kornblum's pressure surface. Another effect is that injection from a double row of holes can result in a thinner cooling film (again implying less dilution) than occurs for single-row injection, since for an equivalent mass rate of coolant injection per unit span the velocity ratios are halved by the use of a second row of holes. Third, it must be acknowledged that the streamwise development of the boundary layer is generally not the same along the pressure side of a turbine blade as along a concave wall of constant curvature.

The convex-wall data of Schwarz are replotted in Figure 9 using the more usual, two-axis format. In combining the blowing rate and the dimensionless streamwise coordinate into the single parameter, $\zeta$, the sense of order inherent in Figure 5 is lost. When injection is three-dimensional (in other words, for discrete-holes injection), the effects of $x / s$ and $M$ simply do not collapse in terms of a single parameter such as $\zeta$. The problem, of course, is the occurrence of jet lift-off: effectiveness data which rise then fall with blowing rate cannot be expected to match well against an equation which predicts the effectiveness to increase steadily with blowing rate (a second problem is that the measured effectiveness can rise then fall in the stream-wise direction, whereas the slot-plate correlation predicts a steady decrease in effectiveness in the streamwise direction). Three-axis plots such as presented here can be more appropriate in describing discrete-holes film cooling results.

\section{CONCLUSION}

A major limitation of three-dimensional plots is that it is difficult to read precise coordinates from the resulting projections; presumably this is why they do not find extensive use. To grasp the overall behavior of particular film cooling schemes, however, three-dimensional plots can be quite helpful. By comparing in this way the results of several film cooling studies, not only are the effects of density ratio, hole spacing, and surface curvature clearly illustrated, but the slot-plate prediction/correlation of a two-dimensional simplified model is also seen to make an 
excellent standard for comparison of a wide variety of film cooling schemes.

\section{Acknowledgments}

Support provided by the U.S. Air Force Office of Scientific Research is greatly appreciated, with special thanks to Major Dan Fant.

\section{Nomenclature}

$D \quad$ diameter of injection hole [m]

$M \quad$ blowing rate, $\rho_{2} u_{2} / \rho_{\infty} u_{\infty}$

$K \quad$ acceleration parameter, $\left(\mu_{\infty} / \rho_{\infty} u_{\infty}^{2}\right) d u_{\infty} / d x$

$\dot{m}_{2} \quad$ mass flow rate of injectant per unit span $[\mathrm{kg} / \mathrm{ms}]$

$\dot{m}_{\infty} \quad$ mass rate of entrainment per unit span of freestream fluid into cooling film $[\mathrm{kg} / \mathrm{ms}]$

$r \quad$ radius of wall $[\mathrm{m}]$

$\mathrm{Re}_{2} \quad$ injection Reynolds number, $\rho_{2} u_{2} s_{\text {eqv }} / \mu_{2}$

$\operatorname{Re}_{D} \quad$ freestream Reynolds number, $\rho_{\infty} u_{\infty} D / \mu_{\infty}$

$s \quad$ slot width [m]

$s_{\text {eqv }}$ equivalent slot width for discrete-holes injection. (eq.[3]) [m]

$T_{2}$ temperature of injectant $[\mathrm{K}]$

$T_{\infty} \quad$ temperature of freestream $[\mathrm{K}]$

$u_{2}$ average velocity of injection $[\mathrm{m} / \mathrm{s}]$

$u_{\infty} \quad$ freestream velocity $[\mathrm{m} / \mathrm{s}]$

$x \quad$ streamwise coordinate (from point of injection) [m]

\section{Greek letters}

$\mu_{2} \quad$ dynamic viscosity of injectant $[\mathrm{kg} / \mathrm{ms}]$

$\mu_{\infty} \quad$ dynamic viscosity of freestream $[\mathrm{kg} / \mathrm{ms}]$

$\eta \quad$ film cooling effectiveness

$\tilde{\eta} \quad$ laterally averaged film cooling effectiveness

$\rho_{2} \quad$ density of injectant $\left[\mathrm{kg} / \mathrm{m}^{3}\right]$

$\rho_{\infty} \quad$ density of freestream $\mathrm{kg} / \mathrm{m}^{3}$

$\zeta$ slot-injection correlation parameter (eq. [2a])

\section{References}

Goldstein, R. J., 1971. Film Cooling, in Advances in Heat Transfer, Ed. T. Irvine and Harnett, J., Vol. 7, pp. 321-379, Academic Press, New York.

Goldstein, R. J., Kornblum, Y., and Eckert, E. R. G., 1982. Film Cooling Effectiveness on a Turbine Blade, Israel Journal of Technology, Vol. 20, pp. 193-200.

Ito, S., 1973. Film Cooling and Aerodynamic Loss in a Gas Turbine Cascade, Ph.D. Thesis, University of Minnesota, Minneapolis.

Ito, S., Goldstein, R. J., and Eckert, E. R. G., 1978. Film Cooling of a Gas Turbine Blade, Journal of Engineering for Power, Vol. 100, No. 3, pp. 476-481.

Jabbari, M. Y., 1973. Film Cooling and Heat Transfer with Air Injection through a Staggered Row of Holes into an Accelerating Flow, Ph.D. Thesis, University of Minnesota, Minneapolis.

Jabbari, M. Y., and Goldstein, R. J., 1978. Adiabatic Wall Temperature and Heat Transfer Downstream of Injection through Two Rows of Holes, Journal of Engineering for Power, Vol. 100, No. 2, pp. 303-307.

Kornblum, Y., 1981. Film Cooling on a Turbine Blade Following Injection through Two Rows of Staggered Holes, M. S. Thesis, University of Minnesota, Minneapolis.

Meroney, R. N., and Bradshaw, P., 1975. Turbulent Boundary-Layer Growth over a Longitudinally Curved Surface, American Institute of Aeronautics and Astronautics Journal, Vol. 13, No. 11, pp. 1448-1453.

Pedersen, D. R., 1971. "Effect of Density Ratio on Film Cooling Effectiveness for Injection through a Row of Holes and for a Porous Slot," $\mathrm{Ph} . \mathrm{D}$. Thesis, University of Minnesota, Minneapolis.

Pedersen, D. R., Eckert, E. R. G., and Goldstein, R. J., 1977. Film Cooling with Large Density Differences between the Mainstream and Secondary Fluid Measured by the Heat-Mass Transfer Analogy, Journal Heat Transfer, Vol. 99, pp. 620-627.

Schwarz, S. G., 1986. Film Cooling of Curved Surfaces, Ph.D. Thesis, University of Minnesota, Minneapolis.

Schwarz, S. G., Goldstein, R. J., and Eckert, E. R. G., 1991. The Influence of Curvature on Film Cooling Performance, Journal of Turbomachinery, Vol. 100, No. 3, pp. 472-478. 

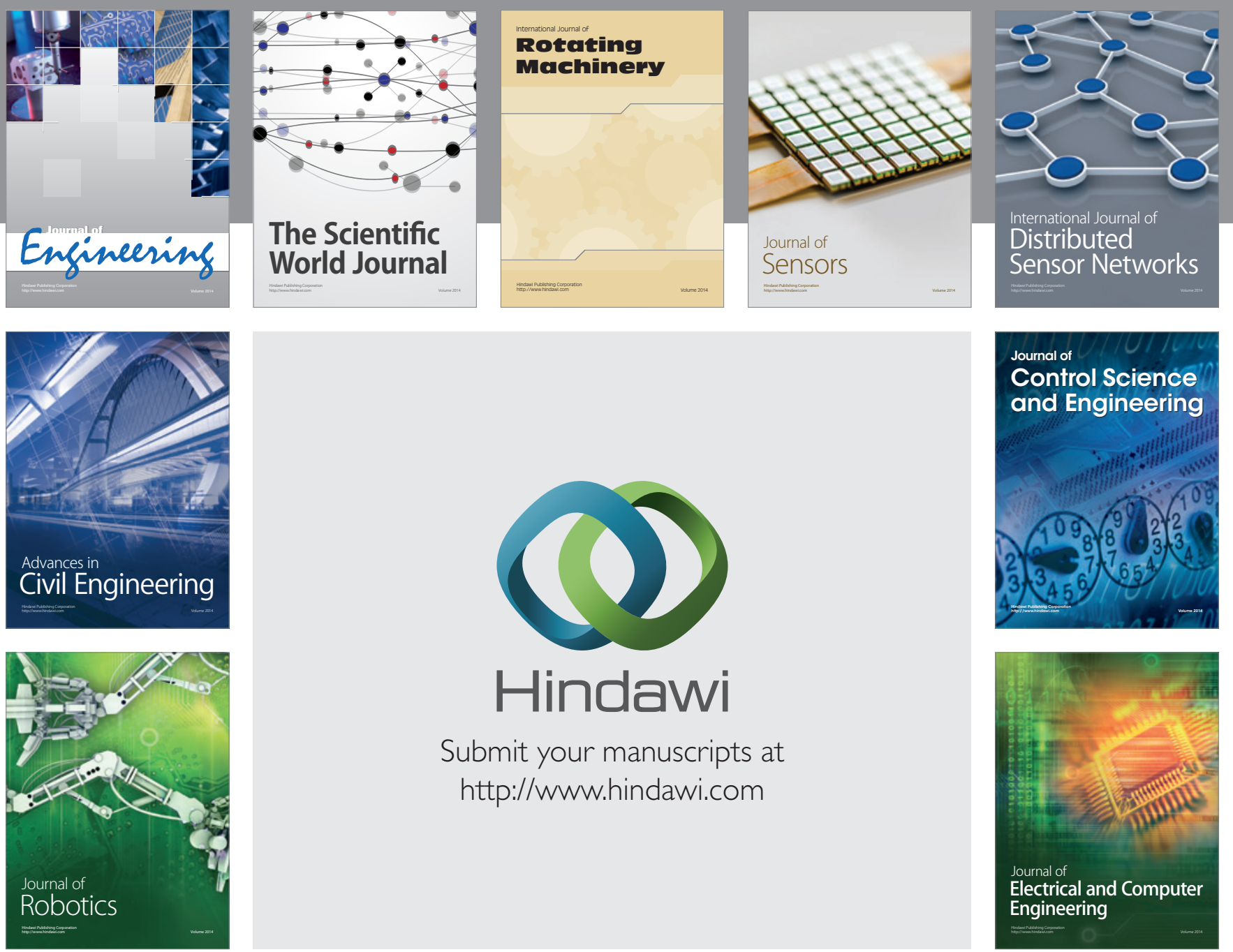

Submit your manuscripts at

http://www.hindawi.com
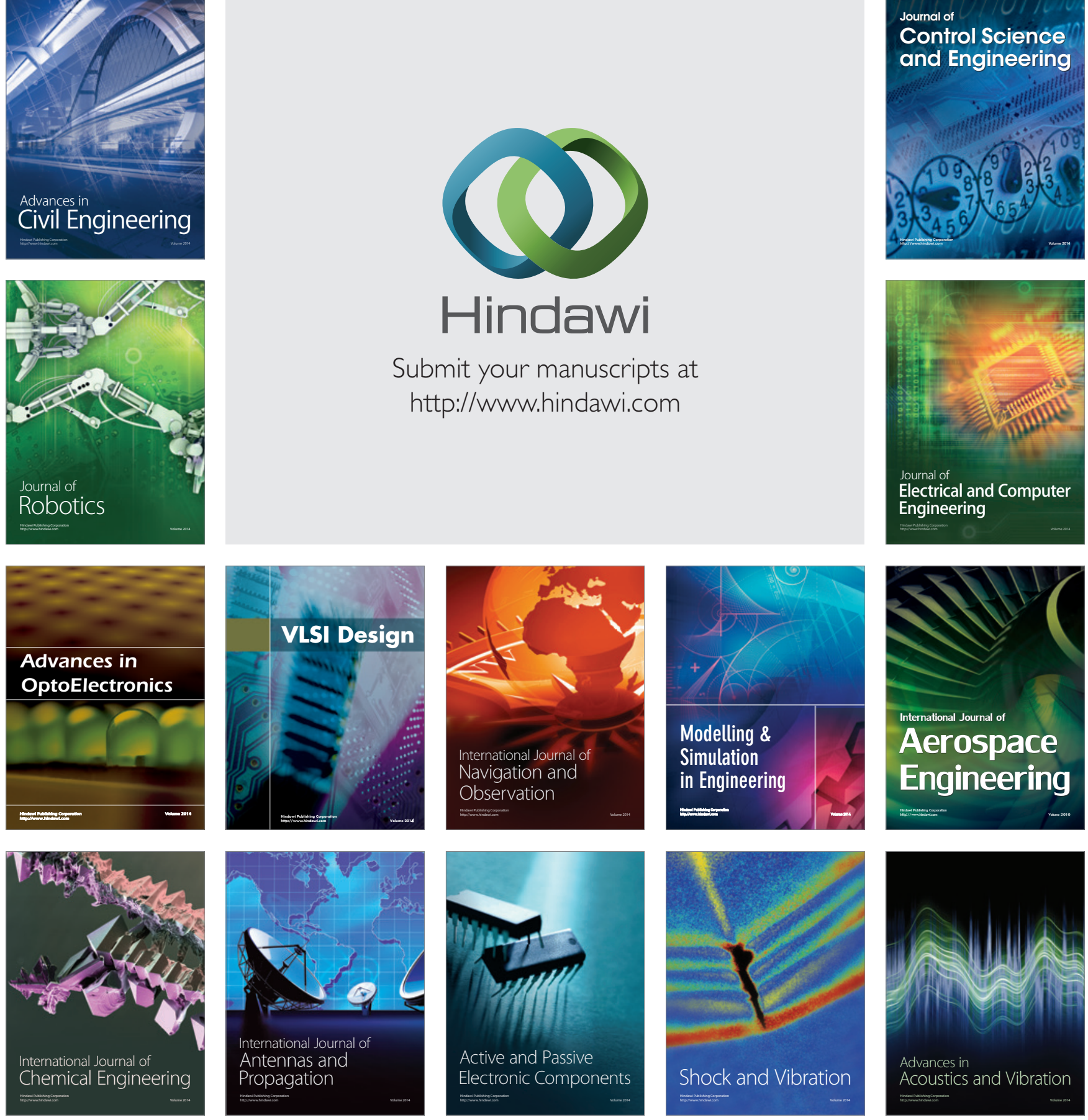